\title{
Separation of Reflection from Diffraction Events via the CRS Technique and a Blind Source Separation Method based on Sparsity Maximization
}

\author{
Leonardo T. Duarte, Renato R. Lopes, Jorge H. Faccipieri, João Marcos T. Romano, Martin Tygel (UNICAMP, Brazil)
}

Copyright 2013, SBGf - Sociedade Brasileira de Geofísica.

This paper was prepared for presentation at the $13^{\text {th }}$ International Congress of the Brazilian Geophysical Society, held in Rio de Janeiro, Brazil, August 26-29, 2013.

Contents of this paper were reviewed by the Technical Committee of the $13^{\text {th }}$ International Congress of The Brazilian Geophysical Society and do not necessarily represent any position of the SBGf, its officers or members. Electronic reproduction or storage of any part of this paper for commercial purposes without the written consent of The Brazilian Geophysical Society is prohibited.

\section{Abstract}

Recent work has shown that, besides providing a conventional stacked section in which reflections are enhanced, the Common-Reflection-Surface (CRS) stacking method may be used to obtain a stacked section mainly composed of diffraction events. Therefore, CRS can be applied to separate reflection and diffraction events in the resulting zero-offset (ZO) sections. However, in practice, there is still mutual interference in the sections obtained by the stacking process. To improve the quality of separation, we propose an additional step based on a signal processing framework known as blind source separation (BSS). The adopted BSS method relies on the concept of sparsity, which suits well the overall characteristic of the reflection events. Numerical experiments with synthetic and field data show that the proposed additional step leads to better results with respect to the stacked section generated by CRS.

\section{Introduction}

The Common-Reflection-Surface (CRS) method (Jäger et al., 2001) is an interesting alternative to the wellestablished Common-midpoint (CMP) stacking. By considering a greater number of traces than those present in a CMP gather, CRS provides, also by means of stacking, a simulated zero-offset (ZO) section with higher signal-to-noise ratio (SNR) as compared to the CMP method (Hertweck et al., 2007).

In addition to increasing the SNR of the simulated ZO section, CRS has also some other interesting features. The CRS stacking moveout depends on a number parameters that are useful for several imaging problems. Such parameters include the usual normal-moveout (NMO)velocity, which is the single parameter used in the CMP method. A particular application of the CRS parameters is the so-called Normal-Incidence-Point (NIP) tomography (Duveneck, 2004).

Recent work (Asgedom et al., 2011; Faccipieri, 2012) has shown that a slight modification on the particular choice of the CRS parameters, can be useful in the task of separating reflections and diffraction events in the $\mathrm{ZO}$ section. Indeed, while the standard CRS stacking leads to an enhancement of reflection events while attenuating diffractions (conventional stacking), a diffraction-modified CRS moveout enables to enhance diffraction events while attenuating reflections (Faccipieri, 2012). This feature is quite attractive, since obtaining a stacked diffraction section is important when imaging geological structures such as faults and pinchouts. Moreover, a section free of diffraction events is required in the tomographic process.

As will be shown later, the stacked section obtained by the traditional CRS may contain residual diffractions. Likewise, the stacked section with enhanced diffractions may contain residual reflections. Put differently, a strategy based only on traveltime stacking may not be enough to develop a sound method to separate diffractions from reflections. To overcome such limitation, an additional signal-processing step is proposed, so that the separation between reflections and diffractions is improved. More precisely, we formulate the mutual interference problem that takes place in the CRS-stacked sections as a blind source separation (BSS) problem (Romano et al., 2011; Comon and Jutten, 2010). In order to tackle the resulting BSS problem, we consider a recently proposed algorithm (Duarte et al., 2011a) that exploits the information that one source, in our case the reflections, is sparse and, furthermore, sparser than the other source (diffractions).

The paper is organized as follows. In the next section we provide a brief review of the CRS stacking technique, followed in the subsequent section by an introduction to the BSS problem and the adopted method. Then, numerical experiments with synthetic and real data are carried out and discussed. Finally, we present our conclusions and perspectives for future work.

\section{The CRS Stacking Method}

The CRS method (Jäger et al., 2001) is a stacking technique with some important differences when compared to the traditional CMP method (Mayne, 1962). For instance, the CRS method performs stacking using not only traces belonging to the CMP of interest, as is done in the CMP method, but also traces that belong to neighbouring CMPs (supergather). To combine these traces appropriately, the CRS method relies on the CRS traveltime, which, for 2D data, is given by

$$
t_{\mathrm{CRS}}\left(h, m_{\mathrm{d}}\right)=\left(t_{0}+a m_{\mathrm{d}}\right)^{2}+b m_{\mathrm{d}}^{2}+c h^{2} .
$$

Here, $t_{0}$ is the two-way zero-offset (ZO) traveltime, $h$ is the trace offset, $m_{\mathrm{d}}=m-m_{0}$ is the trace midpoint displacement, i.e., the difference between the trace midpoint, $m$, and the midpoint in which we are stacking, $m_{0}$. The parameters $a$ and $b$ are related, respectively, to the dip and the curvature of the reflector, while $c$ is related to the normal moveout (NMO) velocity (Jäger et al., 2001). 
Note that the NMO traveltime is given by $t_{\mathrm{NMO}}(h)=$ $t_{\mathrm{CRS}}(h, 0)$, so that the NMO traveltime depends only on the offset, and not the midpoint displacement ${ }^{1}$. Also note that the CRS traveltime depends on three parameters, while the NMO traveltime depends on only one. Finally, we note that there is a $3 \mathrm{D}$ version of the CRS traveltime. Even though all the techniques discussed in this paper can be extended to 3D data, we will not discuss this case in this paper, since the notation for the $2 \mathrm{D}$ case is much simpler.

\section{CRS for Diffractions}

When both reflections and diffractions are present, the CRS method tends to favour the reflections, as their amplitudes are in general larger than those of the diffractions. Still, even though the resulting stacked image contains mostly reflection, it may contain some residual diffractions. On the other hand, some works (Asgedom et al., 2011; Faccipieri, 2012) have proposed methods to generate images with enhanced diffractions. For instance, in (Zhang et al., 2001) it is shown that, when the reflector shrinks to a point, then its curvature is such that the parameter $b$ gets closer and closer to $c$. Thus, a good approximation to the diffraction traveltime is given by making $b=c$, i.e..

$$
t_{\mathrm{D}}=\left(t_{0}+a m_{\mathrm{d}}\right)^{2}+c\left(m_{\mathrm{d}}^{2}+h^{2}\right) .
$$

Direct use of this traveltime to generate an image with diffractions does not generate good results. (Faccipieri, 2012), however, shows that using $b=\varepsilon c$, where $\varepsilon$ is a small perturbation close to 1 , significantly improves the result.

In summary, the use of CRS generates an image with mostly reflections, but some residual diffraction events. The method in (Faccipieri, 2012), on the other hand, generates images with mostly diffractions. In the next section, this mutual interference problem will be formulated as a BSS problem, and we shall discuss one BSS method that is particularly well-suited to this application.

\section{Blind Source Separation to Extract Reflections}

As discussed in the last section, CRS provides two stacked sections containing mainly reflection and diffraction events, respectively. Let us denote these sections by the vectors $\mathbf{x}_{1}$ and $\mathbf{x}_{2}$, respectively. In this work, these vectors correspond to the concatenation of all traces present in the stacked section, or in a given window of this section. Therefore, the size of these vectors are given by $N_{t} N_{s}$, where $N_{t}$ and $N_{s}$ denote the number of traces and samples present in the selected window of the stacked section, respectively.

Ideally, $\mathbf{x}_{1}$ and $\mathbf{x}_{2}$ should contain only reflection and diffraction events, respectively. However, in practice, there is always a residual mutual interference, here described through a linear and memoryless model, that is:

$$
\begin{aligned}
& \mathbf{x}_{1}=a_{11} \mathbf{s}_{1}+a_{12} \mathbf{s}_{2} \\
& \mathbf{x}_{2}=a_{21} \mathbf{s}_{1}+a_{22} \mathbf{s}_{2} .
\end{aligned}
$$

Here, $\mathbf{s}_{1}$ and $\mathbf{s}_{2}$ are vectors (known as sources) containing only reflection and diffraction events, respectively. The parameters $a_{i j}$ are unknown and model the interference

\footnotetext{
${ }^{1}$ This makes sense, as the NMO traveltime assumes that all traces have the same midpoint, which coincides with the midpoint of interest.
}

between reflections and diffractions. Under these conditions, our problem can be formulated as follows: given the stacked sections $\mathbf{x}_{1}$ and $\mathbf{x}_{2}$, estimate $\mathbf{s}_{1}$ and $\mathbf{s}_{2}$ without knowing the mixing parameters $a_{i j}$. In signal processing theory, this problem is known as blind source separation (BSS) (Romano et al., 2011; Comon and Jutten, 2010).

The problem of BSS is ill-posed, i.e., it cannot be solved unless additional information on the sources are provided. For instance, in independent component analysis (ICA), which is the most adopted solution to the BSS problem, one assumes that the sources can be modeled as realizations of mutual independent random variables (Comon, 1994). Therefore, since the mixing process makes $\mathbf{x}_{1}$ and $\mathbf{x}_{2}$ dependent variables, ICA tries to separate the sources by recovering the original independence property.

Unfortunately, there is no guarantee that reflection and diffraction events can be modeled as independent sources, thus limiting the application of ICA to our problem. In view of this limitation, we consider a recently introduced BSS method which, instead of focusing on the independence assumption, is tailored to the case where one of the sources is sparser (in a given domain) than the other (Duarte et al., 2011a). In our application, if the sparsity property is exploited in the time domain, the hypothesis made by Duarte et al. (2011a) simply means that one of the sources is less frequent than the other one in the stacked sections - this is usually the case of reflection events (see, for instance Figures 4 and 5).

In order to retrieve the sparsest source, the method proposed in Duarte et al. (2011a) considers a linear extracting model in which the estimated source is given by:

$$
\mathbf{y}=w_{1} \mathbf{x}_{1}+w_{2} \mathbf{x}_{2} .
$$

Since one is interested in extracting the sparsest component, the extracting parameters $w_{1}$ and $w_{2}$ are adjusted so that $\mathbf{y}$ be as sparse as possible. If the sparsity of $\mathbf{y}$ is measured by the $l_{0}$-norm (namely, the number of non-null elements of the vector $\mathbf{y}$ ), then $w_{1}$ and $w_{2}$ can be found by solving the following optimization problem

$$
\min _{w_{1}, w_{2}}\|\mathbf{y}\|_{0}=\left\|w_{1} \mathbf{x}_{1}+w_{2} \mathbf{x}_{2}\right\|_{0}
$$

where $\|\mathbf{y}\|_{0}$ is the $l_{0}$-norm of $\mathbf{y}$. It can be shown that, if $\left\|\mathbf{s}_{1}\right\|_{0}<\frac{1}{2}\left\|\mathbf{s}_{2}\right\|_{0}$, then solving (6) leads to the extraction of the sparsest component $\mathbf{s}_{1}$ (Duarte et al., 2011a).

In practice, since actual sparse signals take most of the time values close to zero, but not necessarily null, the $l_{0^{-}}$ norm cannot be used. To overcome this problem, Duarte et al. (2011b) proposed a method based on a smoothed version of the $l_{0}$-norm. The algorithm developed in Duarte et al. (2011b) is adopted in the present work, and we refer the reader to this paper for further details of this technique. The application of this method provides us a powerful methodology to retrieve the sparsest component, which in our case is the stacked section associated with the reflection events. This feature is exploited in a set of experiments that are conducted in the next section.

\section{Results}

We here consider the application of the BSS discussed in the last section to two different situations. The first one concerns synthetic data with modeled reflection and 
diffraction sections. In the second example our separation method is applied to field data.

\section{Example with Synthetic Data}

Under the consideration of a synthetic model with two horizontal reflectors and six point diffractors, we generated the two synthetic stacked sections depicted in Figures 1 and 2. The section shown in Figure 1 was obtained by a conventional CRS stacked section. We see the two reflection events with higher amplitude as compared with the amplitudes of the six diffraction events. The section of Figure 2 was obtained by the application of diffraction CRS, namely replacing the full CRS moveout (equation 1) by the diffraction CRS moveout (equation 2). We readily see that the resulting section has enhanced diffractions, as well as attenuated reflections. Figures 1 and 2 originate the vectors $\mathbf{x}_{1}$ and $\mathbf{x}_{2}$, respectively.

After applying the BSS method described before, we obtained the image depicted in Figure 3. Note that, by retrieving the sparsest source (reflections), the BSS method was able to provide a section that is almost free of diffractions, which contrasts with the stacked section obtained by the CRS. This result would be particularly interesting in the context of Normal-incidentpoint (NIP) tomography, since the input data to the inversion processing is obtained by picking a set of reflection events (Duveneck, 2004).

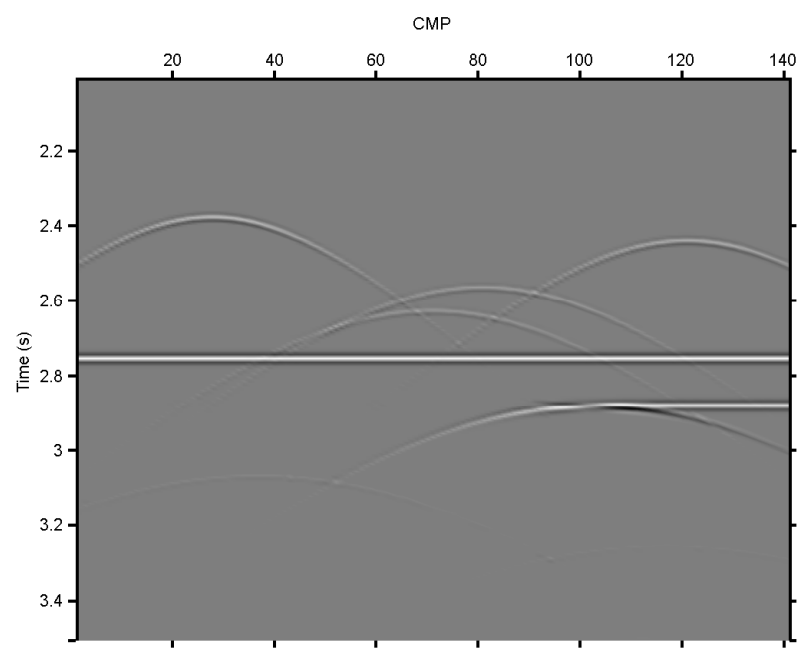

Figure 1: Synthetic data: CRS stacked section (Reflections)

\section{Example with field data}

We now discuss the application of the proposed approach to a real marine dataset acquired over the Jequitinhonha basin (Brazil). The total number of shots was 981 and the temporal sampling rate was $4 \mathrm{~ms}$. Further information of this dataset can be found in Faccipieri (2012). In our experiments, we considered a window of the CRS stacked sections where the mutual interference between reflection and diffraction events was still relevant. These windows are shown in Figures 4 and 5, which correspond to the (reflection enhanced) conventional CRS stacked section (mixture $\mathbf{x}_{1}$ ) and to the (diffraction enhanced) CRS section after CRS diffraction moveout (mixture $\mathbf{x}_{2}$ ), respectively.

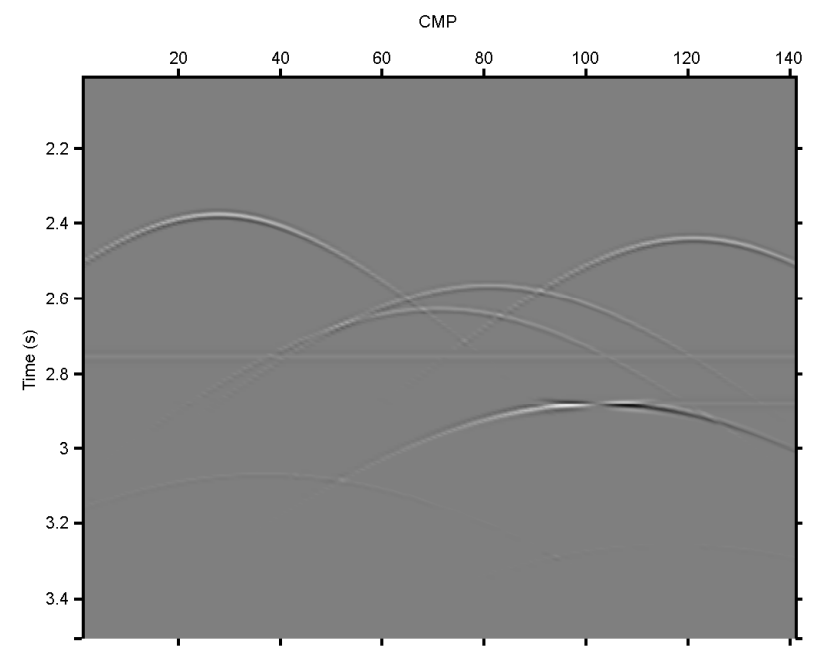

Figure 2: Synthetic data: CRS stacked section (Diffractions)

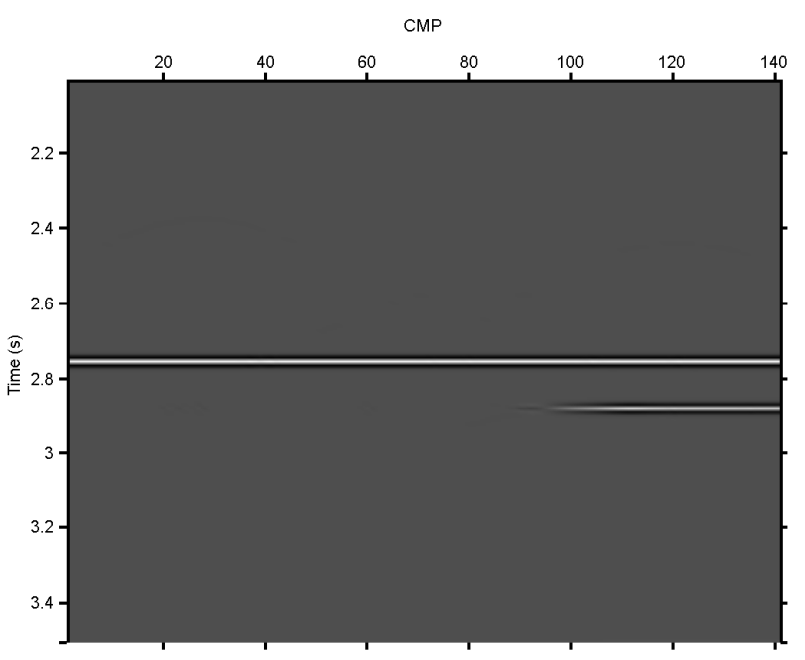

Figure 3: Synthetic data: Retrieved source by BSS method

The application of the BSS method has led to the image depicted in Figure 6. Note that the sparsest source estimated by the BSS method provides a section in which the diffraction events are more attenuated with respect to the original CRS stacked section (Figures 4). This result is even clearer after approximately 2.2s.

\section{Conclusions}

The problem of separating reflection and diffraction events in simulated $\mathrm{ZO}$ sections was addressed based on the conventional CRS stacking (in which reflections are enhanced and diffractions attenuated) and diffraction CRS (in which diffractions are enhanced and reflections attenuated). With the two CRS stacked sections at hand, we set up a signal processing tool to enhance the separation results within the two sections. This task was achieved by a BSS method that basically searches for the sparsest component, here assumed to be associated with the reflection events. Application to synthetic and real data examples confirm that the proposed approach, which exploits the synergy between a geophysical technique and 


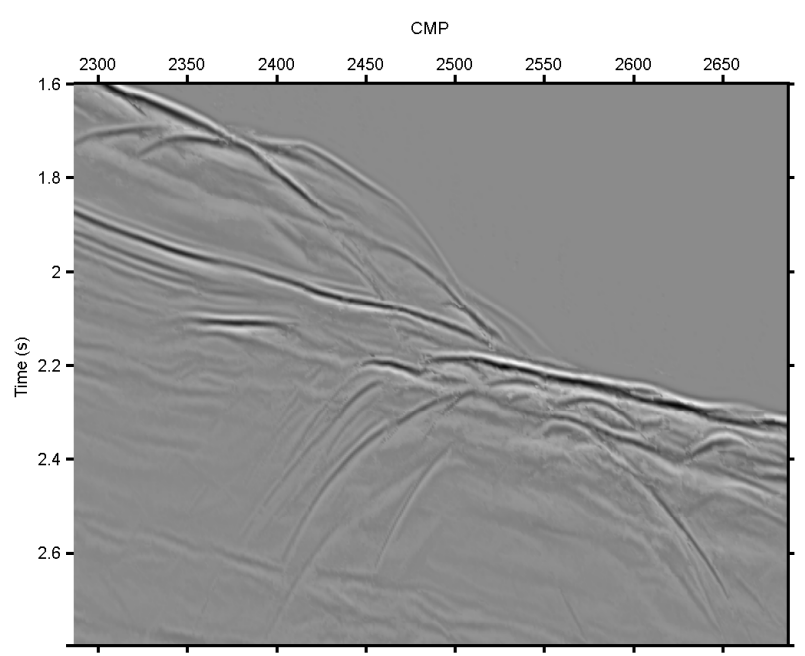

Figure 4: Field data: CRS stacked section (Reflections)

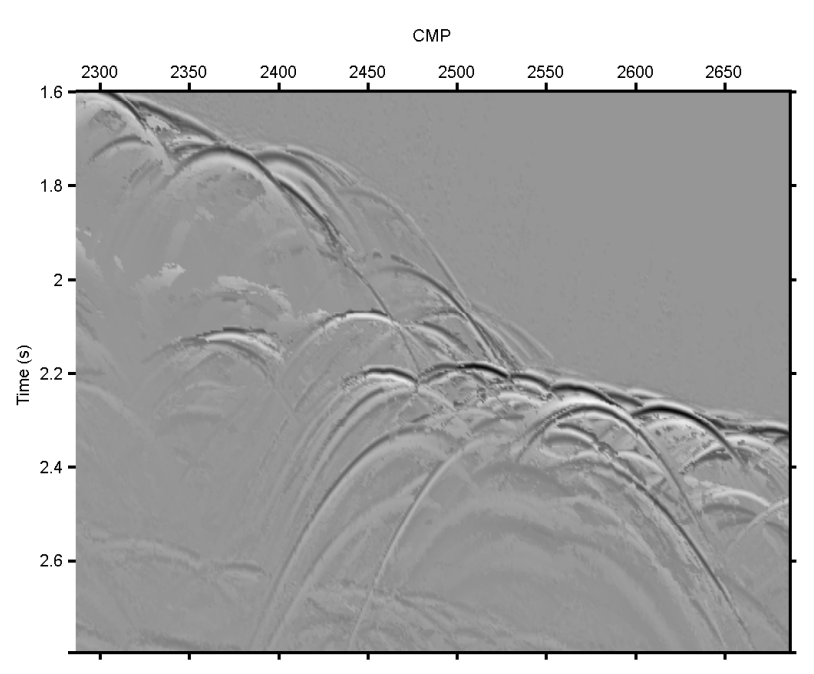

Figure 5: Field data: CRS stacked section (Diffractions)

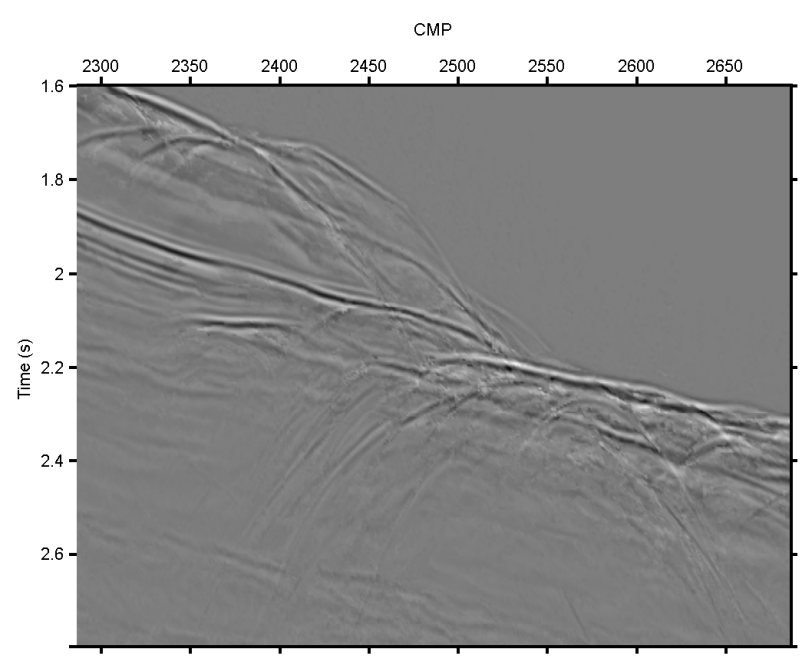

Figure 6: Field data: Retrieved source by BSS method a signal processing tool, can provide a clearer image of the reflection events.

Despite the interesting results obtained in this research, there are several questions that could be addressed in future work. The first one concerns the derivation of a local version of the proposed method. Indeed, in the present work, the BSS method was applied in a global fashion. However, from related problems such as that of multiple attenuation, it is well-known that local approaches may perform better than global approaches (see for instance Donno (2011)). Another topic that could be investigated is the extraction of the component related to the diffraction events. The difficulty here is that the CRS may stack a set of diffractions to explain a reflection event. Finally, one may also envisage an extension of the proposed method to the 3D case.

\section{Acknowledgments}

L. T. Duarte thanks the São Paulo Research Foundation (FAPESP) (Brazil) for financial support. The authors also thank the National Council for Scientific and Technological Development (CNPq-Brazil) also for support. L. T. Duarte, R. R. Lopes and M. Tygel are members of the INCTGP/CNPQ (www.inct-gp.org). Finally, we thank Petrobras for providing the real dataset used in this paper.

\section{References}

Asgedom, E. G., L. J. Gelius, and M. Tygel, 2011, Diffraction separation using the crs technique: A field data application: Presented at the Twelfth International Congress of the Brazilian Geophysical Society (SBGf 2011).

Comon, P., 1994, Independent component analysis, a new concept?: Signal Processing, 36, 287-314.

Comon, P., and C. Jutten, eds., 2010, Handbook of blind source separation: independent component analysis and applications: Academic Press.

Donno, D., 2011, Improving multiple removal using leastsquares dip filters and independent component analysis: Geophyisics, 76, V91-V104.

Duarte, L. T., R. Suyama, R. Attux, J. M. T. Romano, and C. Jutten, 2011a, Blind extraction of sparse components based on 10-norm minimization: Proc. IEEE Statistical Signal Processing Workshop (SSP), 617-620.

$\longrightarrow, 2011 \mathrm{~b}$, Une méthode d'extraction de sources parcimonieuses fondée sur la norme 10: Presented at the Actes du XXIIlième Colloque GRETSI.

Duveneck, E., 2004, Velocity model estimation with dataderived wavefront attributes: Geophyisics, 69, 265-274.

Faccipieri, J. H., 2012, Separaçao e processamento de difrações em dados geofísicos de reflexão: Master's thesis, University of Campinas.

Hertweck, T., J. Schleicher, and J. Mann, 2007, Data stacking beyond cmp: The Leading Edge, 26, 818-827.

Jäger, R., J. Mann, G. HÖcht, and P. Hubral, 2001, Common-reflection-surface stack: image and attributes: Geophysics, 66, 97-109.

Mayne, W. H., 1962, Common reflection point horizontal data stacking techniques: Geophysics, 27, 927-938.

Romano, J. M. T., R. R. F. Attux, C. C. Cavalcante, and R. Suyama, 2011, Unsupervised signal processing: channel equalization and source separation: CRC Press.

Zhang, Y., S. Bergler, and P. Hubral, 2001, Common- 
reflection-surface (crs) stack for common offset: Geophysical Prospecting, 49, 709-718. 